\title{
Avaliando aprendizado de máquina na previsão de curto prazo de séries temporais de energia solar
}

\section{Evaluating machine learning in short-term forecasting time series of solar power}

\author{
Naylene Fraccanabbia ${ }^{1}$ and Viviana Cocco Mariani ${ }^{(0,2}$ \\ ${ }^{1}$ Pontifícia Universidade Católica do Paraná, ${ }^{2}$ Pontifícia Universidade Católica do Paraná \\ *nayfraccanabbia@outlook.com; viviana.mariani@pucpr.br
}

Recebido: 16/05/2021. Revisado: 18/05/2021. Aceito: 28/06/2021.

\begin{abstract}
Resumo
Fontes alternativas de energia estão se tornando cada vez mais frequentes, tendo como objetivo reduzir a poluição ambiental, além de serem ideais para superar a crise energética, logo, neste contexto, a energia solar se destaca por ser abundante. Devido ao alto nível de incerteza dos fatores que interferem diretamente na geração de energia solar, como temperatura e radiação solar, realizar previsões de energia solar com alta precisão é um desafio. Assim, o objetivo deste artigo é desenvolver um modelo de previsão por meio de séries temporais que possibilite prever a produção de energia solar, para 1, 3 e 6 passos à frente, enfatizando a potencialidade da rede neural, utilizando um banco de dados de uma usina fotovoltaica localizada no Uruguai. Para o desenvolvimento da proposta, técnicas de pré-processamento e os métodos de previsão regressão de vetores de suporte (Support Vector Regression, SVR), rede neural perceptron multicamadas com regularização bayesiana (Bayesian Regularized Neural Network, BRNN) e modelo linear generalizado (Generalized Linear Model, GLM) foram combinados. Por fim, tais combinações foram comparadas usando medidas de desempenho. Notou-se que a combinação da análise de componentes principais (Principal Components Analysis - PCA) e a Rede Neural Perceptron Multicamadas com Regularização Bayesiana obteve os melhores resultados, utilizando as três medidas de desempenho.
\end{abstract}

Palavras-Chave: Energia Solar; Previsão de Séries Temporais; Aprendizado de Máquina; Rede Neural Artificia

\begin{abstract}
Alternative sources of energy are becoming more and more frequent, aiming to reduce environmental pollution, besides being ideal to overcome the energy crisis, therefore, in this context, solar power stands out for being abundant. Due to the high level of uncertainty of the factors that directly interfere in the generation of solar power, such as temperature and solar radiation, making solar energy predictions with high precision is a challenge. Thus, the objective of this article is to develop a forecast model through time series that makes it possible to predict the production of solar power, for 1,3 and 6 steps ahead, emphasizing the potential of the neural network, using a database of one photovoltaic plant located in Uruguay. For the development of the proposal, pre-processing techniques and forecasting methods Support Vector Regression (SVR), Bayesian Regularized Neural Network (BRNN) and Generalized Linear Model (GLM) were combined. Finally, these combinations were compared using performance measures. It was noted that the combination of principal components analysis (Principal Components Analysis - PCA) and the Multilayer Perceptron Neural Network with Bayesian Regularization obtained the best results, using the three performance measures.
\end{abstract}

Keywords: Solar Power; Time Series Forecasting; Machine Learning; Artificial Neural Network. 


\section{Introdução}

Atualmente, o consumo de energia é um dos critérios utilizados na análise de desenvolvimento econômico dos países, estando o mesmo em acentuada expansão devido ao avanço econômico e ao aumento da demanda, em especial do mercado de energia renovável (Behera et al., 2018).

Fontes não renováveis de energia, como carvão, petróleo e gás natural, são utilizados em grande escala pela população mundial. Entretanto deve-se considerar que tais combustíveis fósseis podem acabar se esgotando, devido ao aumento da população mundial e desenvolvimento econômico (Zeren and Akkuş, 2020).

Além disso, os combustíveis fósseis prejudicam o meio ambiente, aumentando o aquecimento global, impactando nas condições climáticas e na política econômica do governo (Behera et al., 2018).Devido a fatores como estes, no planeta iniciou-se o desenvolvimento e uso de fontes alternativas de energia limpa e sustentável, com o intuito de reduzir a poluição ambiental e a emissão de $\mathrm{CO} 2$, além de ser uma solução ideal para superar a crise energética (Gouda et al., 2019).

A energia solar é gerada por intermédio da luz do sol, a qual trata-se do recurso natural mais abundante e com maior disponibilidade em todo planeta. Por meio dela, pode-se gerar energia elétrica através da tecnologia fotovoltaica, por exemplo, a qual caracteriza-se pela transformação da radiação solar em energia elétrica solar. Contudo, é importante ressaltar que as fontes de energias renováveis se caracterizam pela sua variabilidade e intermitência, assumindo a necessidade de planejamento em relação ao aumento da participação na matriz energética mundial, e da previsão precisa de fatores como consumo e geração deste recurso (Gemignani, 2018).

O uso efetivo de energias renováveis tem se tornado objeto de pesquisa nas últimas décadas, sendo um dos fatores que contribuí para este crescimento, a necessidade de realizar previsões precisas. Diante disso, pesquisadores adotaram diferentes métodos com o intuito de melhorar a precisão das previsões. Destaca-se a energia eólica e a solar, que atualmente são as fontes renováveis mais usadas e apresentam, nos testes de previsão um desvio relativamente grande entre os resultados previstos e o valor real. Essa dificuldade se deve há alguns fatores que caracterizam as fontes de energia, como a solar por exemplo, que é vulnerável a temperatura, estação do ano, e intensidade da luz. Para tal é relevante explorar algoritmos de aprendizado de máquina que se mostram eficientes para previsões envolvendo grandes quantidades de dados (Persson et al., 2017).

Atualmente, é possível encontrar trabalhos presentes na literatura que buscam melhorar as previsões de energias renováveis explorando algoritmos de aprendizado de máquina. Tal afirmativa pode ser vista nos seguintes trabalhos:

- Previsão de energia solar baseada em métodos de aprendizagem de conjuntos (Fraccanabbia et al., 2020);

- Decomposição híbrida de múltiplos estágios com modelo paramétrico aplicado à previsão da velocidade do vento no Nordeste brasileiro (Moreno et al., 2021);

- Previsão de energia eólica a curto prazo com base no conjunto de empilhamento (Moreno et al., 2019);

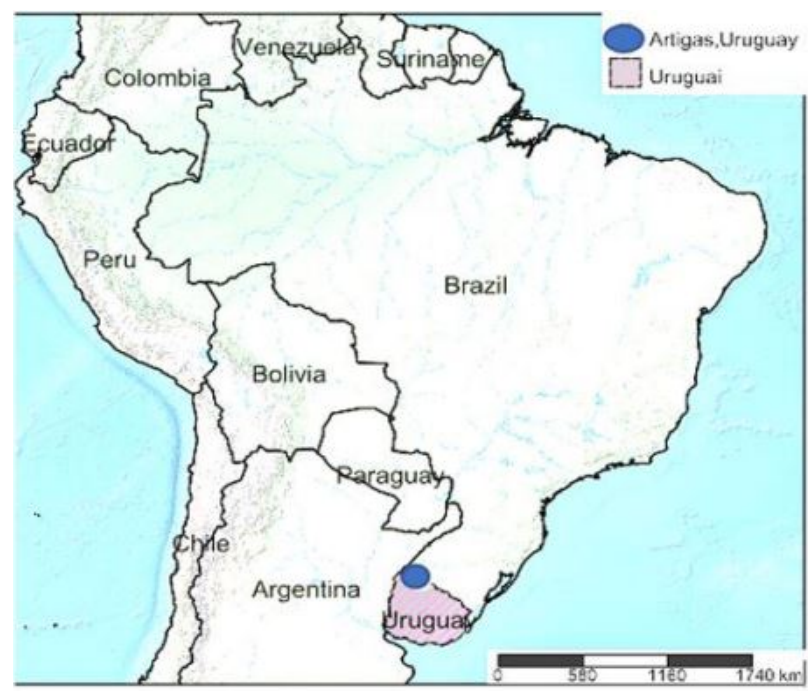

Figura 1: Localização da unidade fotovoltaica.

- Rede Bayesiana de Estado de Eco aplicada à previsão de curto prazo (Trierweiler Ribeiro et al., 2020);

- Estrutura de aprendizado de conjunto de decomposição para previsão de energia eólica em várias etapas (da Silva et al., 2021).

Este trabalho tem como objetivo prever, para $1(10 \mathrm{mi}-$ nutos), 3 (30 minutos) e 6 (60 minutos) passos à frente, por meio de séries temporais, a produção de energia solar. Para tanto, busca-se encontrar o método de aprendizado de máquina mais eficaz, uma vez que, nos últimos tempos, diversas abordagens têm sido propostas com o objetivo de fornecer previsões e oferecer sistemas de apoio à tomada de decisão. Assim, pretende-se analisar qual método apresenta os melhores resultados para estudos deste perfil. De encontro a isso, objetiva-se enfatizar a potencialidade da rede neural.

\section{Materiais e métodos}

\subsection{Material}

O conjunto de dados é de uma planta fotovoltaica de 26,35MWp localizada em Artigas no Uruguai, como pode ser visto na Fig. 1. A escolha por tais dados justifica-se devido a parceria entre um dos funcionários desta empresa e a Pontifícia Universidade Católica do Paraná, visto que o mesmo cursa Pós-Doutorado nesta mesma instituição. O período da amostra utilizada começa em 21 de abril de 2018 às 00 hoo e termina em 23 de abril de 2018 às 23 h50, sendo coletados a cada 10 minutos. A amostra consiste em três variáveis, como mostra a Tabela 1. Na Fig. 2 é possível observar o conjunto de dados dividido em treino e teste.

Tabela 1: Entradas e saída do sistema de energia solar.

\begin{tabular}{lll} 
TIPO & DESCRIÇÃO & UNIDADE DE MEDIDA \\
\hline Entrada & Temperatura & ${ }^{\circ} \mathrm{C}$ \\
Entrada & Radiação & $\mathrm{W} / \mathrm{m} 2$ \\
Saída & Potência & $\mathrm{kW}$ \\
\hline
\end{tabular}




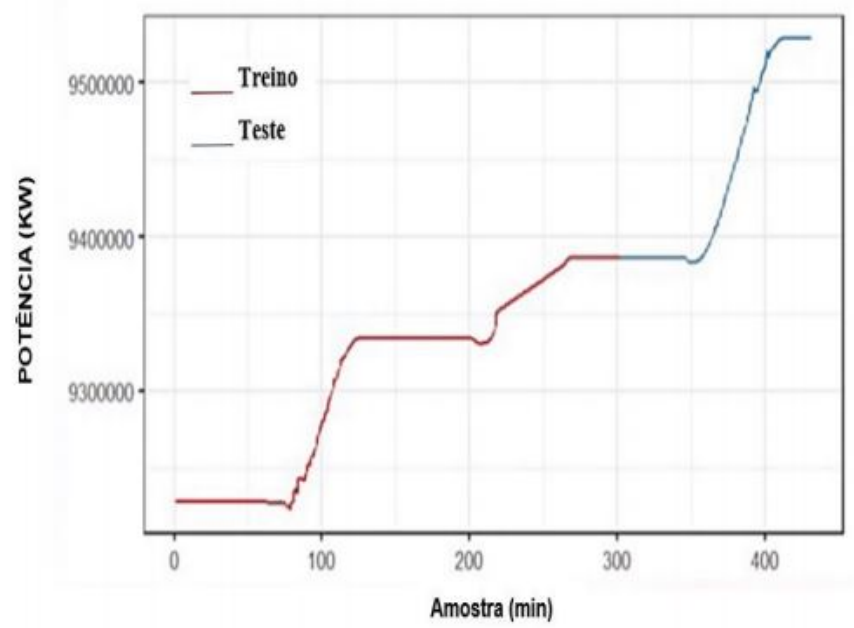

Figura 2: Conjunto de dados dividido em treino e teste, referentes a geração de energia solar (KW), no ano de 2018, em uma planta fotovoltaica localizada em Artigas, no Uruguai.

\subsection{Técnicas de pré-processamento}

As técnicas de pré-processamento visam reduzir a dimensionalidade dos dados.

\subsubsection{Principal Component Analysis}

Principal Component Analysis (PCA), em português Análise de Componentes Principais, tem como ideia principal reduzir a dimensionalidade dos dados que consiste em muitas variáveis correlacionadas entre si. Isso acontece com a transformação de variáveis, em um novo conjunto, que são os componentes principais (Du and Zhu, 2019).

\subsubsection{Correlation Matrix}

Correlation Matrix (CORR) é uma matriz de correlação. Os preditores são pré-processados usando uma matriz de correlação, que remove os preditores correlacionados acima de um limite. Assim, a função CORR calcula correlações entre variáveis preditoras (Kuhn et al., 2013).

\subsection{Métodos}

\subsubsection{Support Vector Regression}

A regressão de vetores de suporte (SVR) com o kernel linear e kernel radial, funciona em princípio semelhante à classificação da máquina de vetores de suporte (SVM). Pode-se dizer que o SVR é a forma adaptada do SVM quando a variável dependente é numérica e não-categórica. As SVMs podem ser caracterizadas como um conjunto de métodos de aprendizado supervisionado relacionados, populares na execução de análises de classificação e regressão. Cada método específico varia de acordo com a estrutura e os atributos do classificador. Mais detalhadamente, o SVM cria um hiperplano ou um conjunto de hiperplanos para classificar todas as entradas em um espaço de alta dimensão (Ring and Eskofier, 2016).A técnica SVR depende das funções do kernel. Existem alguns tipos de kernel, como o linear e o radial.

Em suma, o SVR usa o mesmo princípio que o SVM, mas para problemas de regressão. O SVM linear é um algoritmo eficiente para classificação e regressão em dados estruturados linearmente. Seja $x_{i}, i=1,2, \ldots, n$ e $n$ amostras de treinamento:

$$
f(x)=w^{T} x+b
$$

Onde $x_{i}$ é uma entrada $\mathrm{n}$-dimensional, $w$ é o vetor de peso e $b$ é a tendência. O SVM também pode ser aplicado se um kernel $k$ for empregado, isso para dados não linearmente estruturados. Para tal é necessário um certo número $S$ de vetores de suporte $x_{i}$, além dos coeficientes correspondentes $a_{i} \in R$. Sendo assim, tem-se:

$$
f(x)=\sum_{i=1}^{S} a_{i} k\left(x_{i}, x\right)+b
$$

Portanto, para um SVM do kernel, a função deve ser avaliada para cada nova instância $x$.

\subsubsection{Bayesian Regularized Neural Network}

Uma das maiores dificuldades encontradas ao projetar um modelo de RNA (Rede Neural Artificial), é determinar o número de neurônios ocultos, uma vez que muitos deles podem levar a um ajuste excessivo. É sabido que um modelo de RNA caracterizado por ser muito complexo ou muito simples terá desempenhos preditivos pobres. Assim, a Rede Neural Perceptron Multicamadas com Regularização Bayesiana (Bayesian Regularized Neural Network, BRNN), que pode ser definida como um tipo de rede neural, visa evitar que ajustes excessivos sejam feitos. BRNN consiste em uma entrada e uma camada oculta que usa método de otimização Bayesiano que busca estimar hiperparâmetros, evitando que tais ajustes sejam feitos (MacKay, 1992).

Apresenta-se, como exemplo de modelo de rede neural, a Fig. 3 que representa uma regularização bayesiana em um modelo de rede neural para estimar linhas de código usando pontos de função:

\subsubsection{Generalized Linear Model}

O Modelo Linear Generalizado (Generalized Linear Model, GLM) permite que modelos diferentes e não lineares sejam testados no contexto de regressão. Este modelo é usado em muitas análises estatísticas, como: regressão múltipla e análise de variância. O objetivo do GLM é estender a ideia de modelagem linear para casos para os quais a relação linear entre uma variável independente e a resposta média, enquanto a distribuição normal não é apropriada para a distribuição de erros (Liu and Lu, 2018).

A média, $\mu$, da distribuição depende das variáveis independentes, $\mathrm{X}$, por meio de:

$$
E(\mathbf{Y} \mid \mathbf{X})=\mu=g^{-1}(\mathbf{X} \beta)
$$

em que $\mathrm{E}(\mathrm{Y} \mid \mathrm{X})$ é o valor esperado de $\mathrm{Y}$ condicional em 


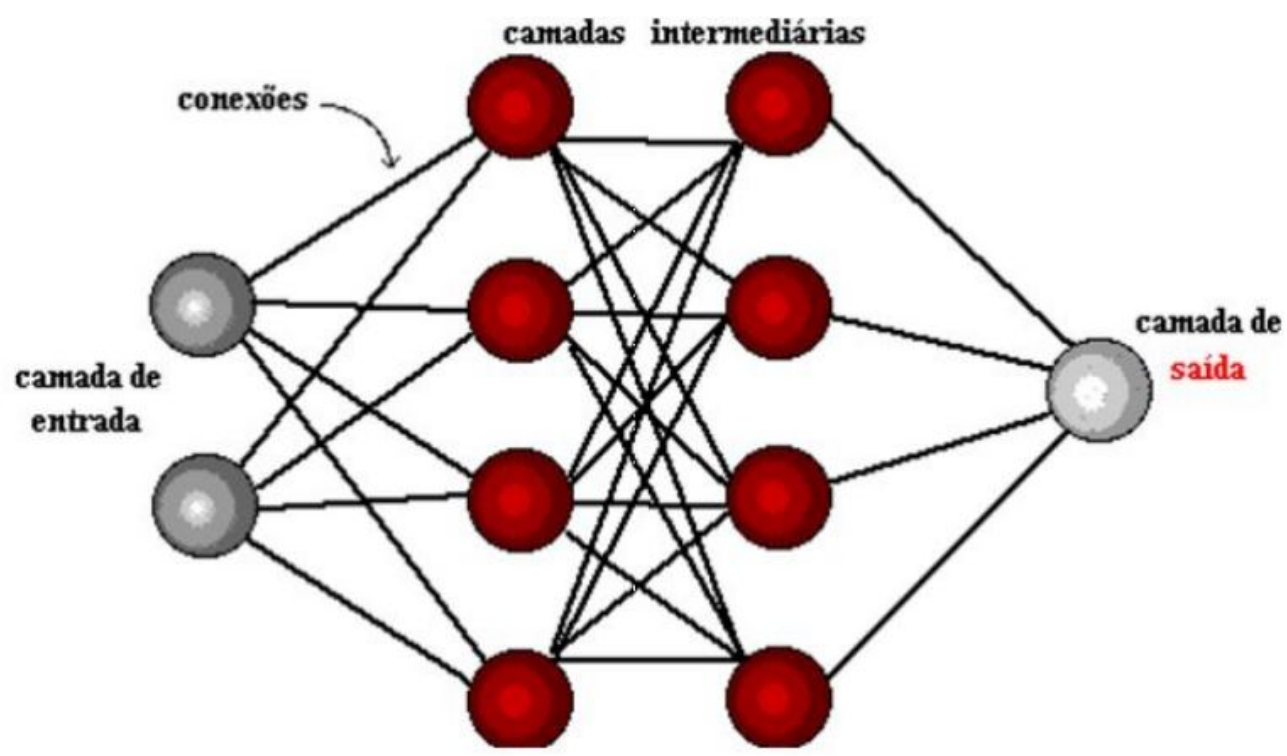

Figura 3: Exemplo de rede neural. Adaptado de: https://sites.icmc.usp.br/andre/research/neural.

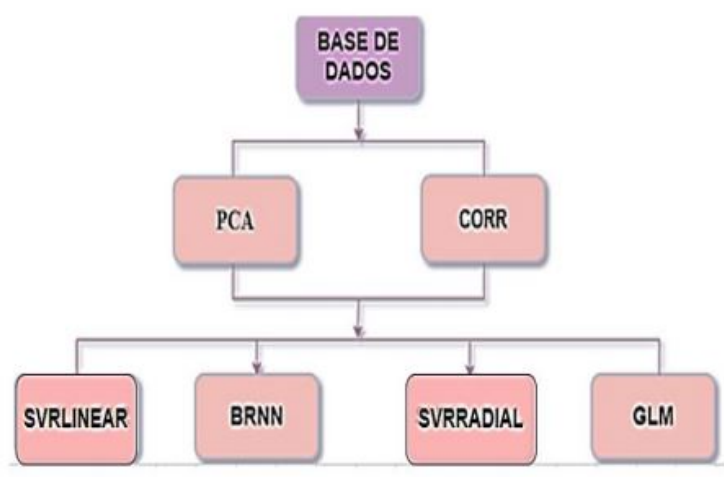

Figura 4: Fluxograma das técnicas aplicadas.

$\mathrm{X} ; \mathrm{X} \beta$ é o preditor linear, uma combinação linear de parâmetros desconhecidos; $g$ é a função de ligação (Nelder and Wedderburn, 1972).

\section{Metodologia}

Esta seção descreve as etapas da metodologia, como ilustra a Fig. 4. Verificou-se por meio das funções de autocorrelação e autocorrelação parcial os atrasos significativos para precisão da previsão. Foi feita a divisão da amostra em treino e teste, sendo no treino 303 observações $(70 \%)$ e no teste 130 observações (30\%). Na simulação foi feita a combinação de cada técnica de pré-processamento com cada modelo utilizado. Assim, foram geradas: PCA-SVML, PCA-BRNN, PCA-SVMR, PCA-GLM, CORR-SVML, CORR-BRNN, CORR-SVMR, CORR-GLM. Para mensuração de performance, foram obtidas as medidas de desempenho: Relative Root Mean Square Error: RRMSE, em português erro relativo ao quadrado médio da raiz, é um indicador que é calculado dividindo o RMSE pelo valor médio dos dados medidos (Despotovic et al., 2016); Determination Coefficient: $R^{2}$, em português coeficiente de determinação, pode ser considerado um coeficiente de correlação múltipla, ou seja, a correlação entre a variável dependente e o conjunto de variáveis independentes (Takeda and Kanamori, 2014). Mean Absolute Percentage Error: MAPE, em português erro absoluto médio percentual, mede o erro em porcentagem, sendo calculado como a média do erro percentual (Goodwin and Lawton, 1999).

\section{Resultados e discussões}

Tendo como objetivo o desenvolvimento de um modelo de previsão que possibilitasse o conhecimento de valores futuros da produção de energia solar, visando prever um, três e seis passos à frente, foram utilizados algoritmos para análise e técnicas de pré-processamento. Ao analisar qual método apresenta os melhores resultados para o presente estudo, foi possível obter os resultados apresentados nas Tabelas 3 e 4 que descrevem as medidas de desempenho dos modelos, tanto para um, quanto para três e seis passos à frente. E nas Figs. 5 e 6, que comparam os valores observados com os preditos, respectivamente, da previsão que obteve os melhores resultados, sendo ela a previsão de um passo à frente. Também, nas Figs. 7 a 10, são apresentados os melhores resultados para três e seis passos à frente. Os melhores resultados estão destacados em negrito. As medidas de desempenho aplicadas foram MAPE, RRMSE e $R^{2}$.

Para determinar qual modelo de previsão proposto é 
mais adequado para cada estudo de caso investigado neste trabalho, verificam-se os critérios apresentados na Tabela 2 (He et al., 2018, Despotovic et al., 2016):

Tabela 2: Critérios RRMSE e MAPE

\begin{tabular}{llll}
\hline RRMSE $(\%)$ & Poder de previsão & MAPE $(\%)$ & Poder de previsão \\
\hline$<10$ & Excelente & $<10$ & Excelente \\
$10-20$ & Bom & $10-20$ & Bom \\
$20-30$ & Razoável & $20-50$ & Razoável \\
$>30$ & Incorreto & $>50$ & Incorreto
\end{tabular}

Já no caso da medida de desempenho $R^{2}$ seu valor fica normalmente entre 0 e 1 , e quanto maior o valor, melhor a concordância entre modelo e observação. Analisando a Tabela 3, que apresenta os melhores resultados, e as Figs. 5 e 6 nota-se que a combinação PCA - BRNN obteve os melhores resultados, para as três medidas de desempenho, o que vem de encontro ao exposto nos objetivos, a potencialidade da rede neural que apresentou resultados excelentes. Salienta-se que as simulações foram feitas com o software RStudio, o qual caracteriza-se como um software livre de ambiente de desenvolvimento integrado para $\mathrm{R}$, uma linguagem de programação para gráficos e cálculos estatísticos.

Tabela 3: Medidas de desempenho obtidas para a previsão de 1 passo à frente (10 minutos).

\begin{tabular}{llll}
\hline & MAPE & RRMSE & $R^{2}$ \\
\hline PCA-SVRLINEAR & 0,0574 & 0,0599 & 0,9852 \\
PCA-BRNN & $\mathbf{0 , 0 0 5 0}$ & $\mathbf{0 , 0 0 6 9}$ & $\mathbf{0 , 9 9 8 4}$ \\
PCA-SVRRADIAL & 0,2404 & 0,2458 & 0,4225 \\
PCA-GLM & 0,0613 & 0,0632 & 0,9503 \\
\hline CORR-SVRLINEAR & 0,1386 & 0,1464 & 0,9148 \\
CORR-BRNN & 0,0078 & 0,0092 & 0,9916 \\
CORR-SVRRADIAL & 0,2268 & 0,2447 & 0,1182 \\
CORR-GLM & 0,1398 & 0,1487 & 0,9652 \\
\hline
\end{tabular}

Tabela 4: Medidas de desempenho obtidas para a previsão de 3 passos à frente (30 minutos).

\begin{tabular}{llll}
\hline & MAPE & RRMSE & $R^{2}$ \\
\hline PCA-SVRLINEAR & 0,0675 & 0,0601 & 0,9744 \\
PCA-BRNN & $\mathbf{0 , 0 0 5 8}$ & $\mathbf{0 , 0 0 7 0}$ & $\mathbf{0 , 9 9 8 1}$ \\
PCA-SVRRADIAL & 0,3564 & 0,2624 & 0,4102 \\
PCA-GLM & 0,0723 & 0,0712 & 0,9455 \\
\hline CORR-SVRLINEAR & 0,1458 & 0,1399 & 0,9056 \\
CORR-BRNN & 0,0069 & 0,0088 & 0,9901 \\
CORR-SVRRADIAL & 0,2357 & 0,2551 & 0,1245 \\
CORR-GLM & 0,1459 & 0,1621 & 0,9563 \\
\hline
\end{tabular}

\section{Conclusões}

Este estudo foi desenvolvido com o objetivo de prever a produção de energia solar, utilizando um banco de dados coletados em uma planta fotovoltaica localizada no Uruguai. Fez-se uso de dados referentes à energia solar devido à crescente demanda pelo uso de energia renovável e por

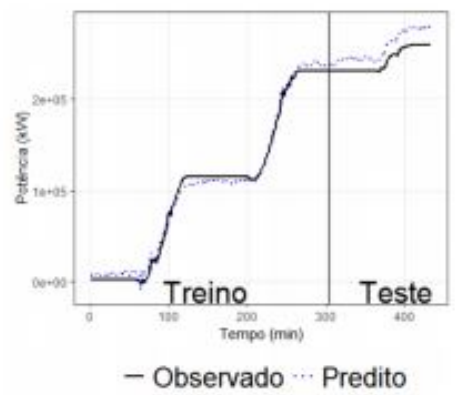

(a)

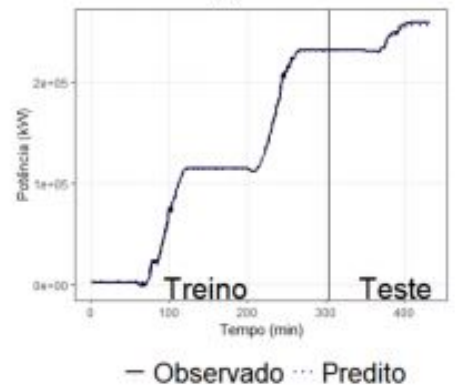

(b)

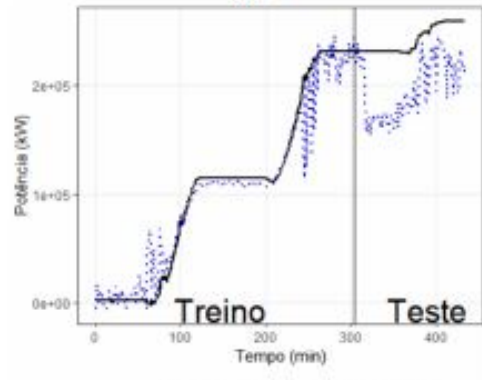

- Observado … Predito

(c)

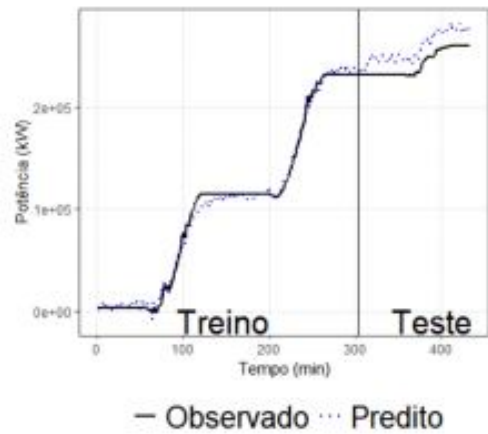

(d)

Figura 5: Valores observados e preditos para previsão de 1 passo à frente aplicando (a) PCA-SVR linear, (b) PCA-BRNN, (c) PCA-SVR Radial; (d) PCA-GLM.

ser um recurso natural disponível em todo planeta.

O formato de uma curva de irradiância fotovoltaica diária pode ser visto como uma meia senoide, e a curva de produção de potência, caso não tenha problemas de co- 


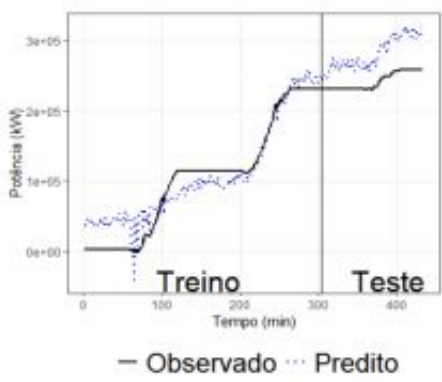

(a)

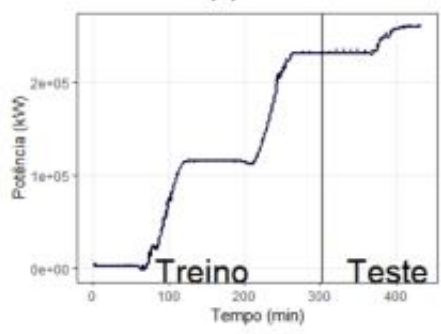

- Observado … Predito

(b)

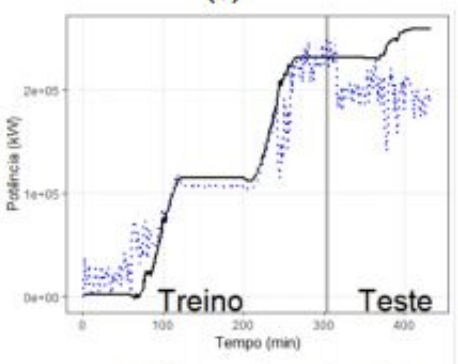

- Observado … Predito

(c)

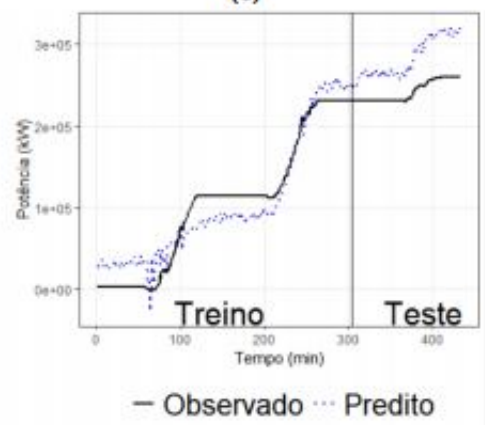

(d)

Figura 6: Valores observados e preditos para previsão de 1 passo à frente aplicando (a) CORR-SVR linear, (b) CORR-BRNN, (c) CORR-SVR Radial; (d) CORR-GLM.

bertura por nuvens (ou desligamento da planta solar por outro motivo elétrico/sistêmico), tem a mesma envoltória, ou seja, um semi-ciclo de senoide. O processamento de dados realizado para tornar essa meia senoide em valores crescentes de potência foi uma soma cumulativa dos dados amostrados a cada período de tempo.

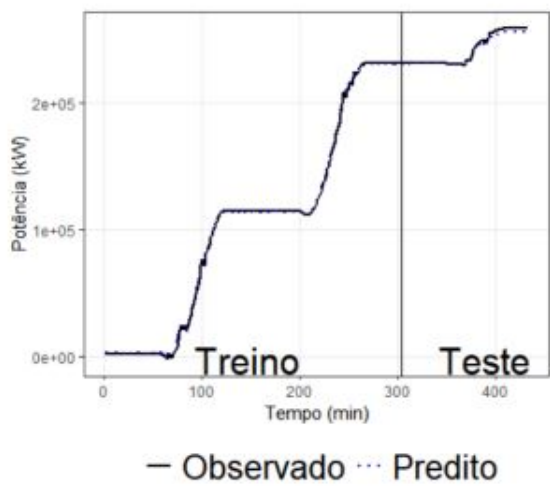

Figura 7: Melhor resultado obtido, para valores observados e preditos para previsão de 3 passos à frente, aplicando CORR-BRNN.

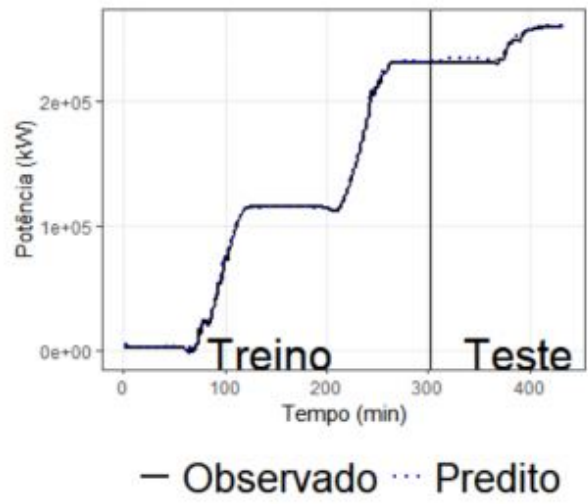

Figura 8: Melhor resultado obtido, para valores observados e preditos para previsão de 6 passos à frente, aplicando PCA-BRNN.

Para o desenvolvimento da proposta, técnicas de préprocessamento combinadas com modelos de previsão foram comparados usando as medidas de desempenho. Por fim, nota-se que a combinação PCA-BRNN obteve os melhores resultados. Além disso percebe-se que ao aumentar o número de passos à frente os resultados acabam se tornando menos satisfatórios, o que se deve ao fato de que quanto maior for o período de tempo desta previsão, maior será o desafio de encontrar métodos e técnicas capazes de aprender e prever com os dados utilizados. Ou seja, os melhores resultados foram obtidos na previsão de um passo à frente.

Para pesquisas futuras, propõe-se aumentar o número dos passos à frente e aplicar testes estatísticos, objetivando cada vez mais uma melhor acurácia nas previsões. 


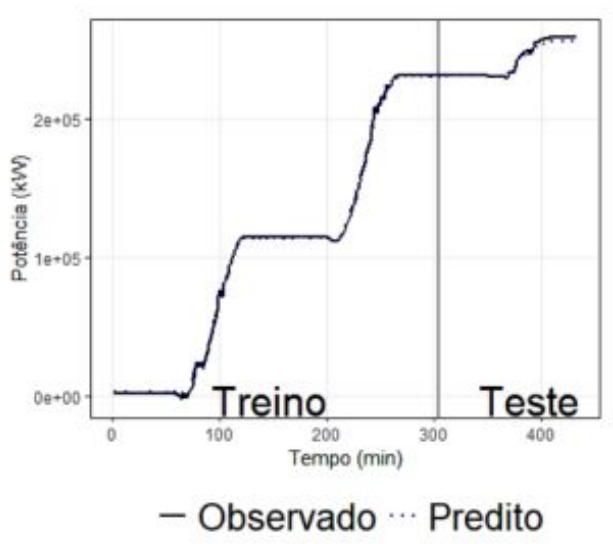

Figura 9: Melhor resultado obtido, para valores observados e preditos para previsão de 6 passos à frente, aplicando CORR-BRNN.

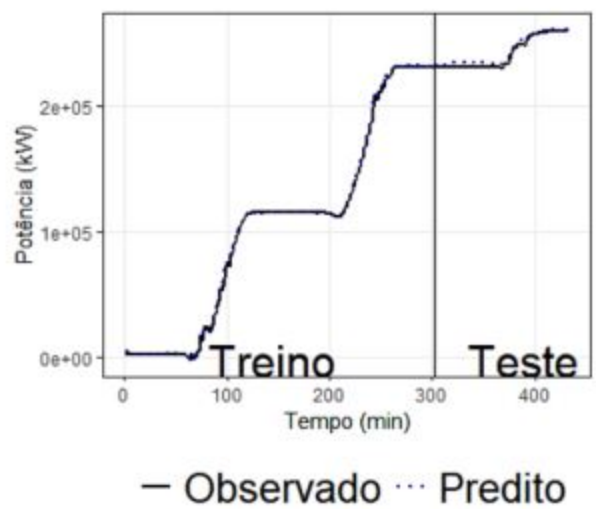

Figura 10: Melhor resultado obtido, para valores observados e preditos para previsão de 6 passos à frente, aplicando PCA-BRNN.

\section{Referências}

Behera, M. K., Majumder, I. and Nayak, N. (2018). Solar photovoltaic power forecasting using optimized modified extreme learning machine technique, Engineering Science and Technology, an International Journal 21(3): 428-438. https://doi.org/10.1016/j.jestch. 2018.04.013.

da Silva, R. G., Ribeiro, M. H. D. M., Moreno, S. R., Mariani, V. C. and dos Santos Coelho, L. (2021). A novel decomposition-ensemble learning framework for multi-step ahead wind energy forecasting, Energy 216: 119174. https://doi.org/10.1016/j.energy. 2020. 119174 .

Despotovic, M., Nedic, V., Despotovic, D. and Cvetanovic, S. (2016). Evaluation of empirical models for predicting monthly mean horizontal diffuse solar radiation,
Renewable and Sustainable Energy Reviews 56: 246-260. http://dx.doi.org/10.1016/j.rser.2015.11.058.

Du, X. and Zhu, F. (2019). A novel principal components analysis (pca) method for energy absorbing structural design enhanced by data mining, Advances in Engineering Software 127: 17-27. https://doi . org/10.1016/j . advengsoft.2018.10.005.

Fraccanabbia, N., da Silva, R. G., Henrique Dal Molin Ribeiro, M., Moreno, S. R., dos Santos Coelho, L. and Mariani, V. C. (2020). Solar power forecasting based on ensemble learning methods, 2020 International Joint Conference on Neural Networks (IJCNN), pp. 1-7. https : //doi.org/10.1109/IJCNN48605.2020.9206777.

Gemignani, M. M. F. (2018). Cenários sintéticos de radiação solar para estudos energéticos., $\mathrm{PhD}$ thesis, Universidade de São Paulo.

Goodwin, P. and Lawton, R. (1999). On the asymmetry of the symmetric mape, International journal of forecasting 15(4): 405-408. https://doi.org/10.1016/ S0169-2070 (99) 00007-2.

Gouda, S. G., Hussein, Z., Luo, S. and Yuan, Q. (2019). Model selection for accurate daily global solar radiation prediction in china, Journal of cleaner production 221: 132144. https://doi .org/10.1016/j. jclepro.2019.02.211.

He, Q., Wang, J. and Lu, H. (2018). A hybrid system for short-term wind speed forecasting, Applied energy 226: 756-771. https://doi.org/10.1016/j.apenergy . 2018.06 .053$.

Kuhn, M., Johnson, K. et al. (2013). Applied predictive modeling, Vol. 26, Springer.

Liu, X.-W. and Lu, D.-G. (2018). Survival analysis of fatigue data: Application of generalized linear models and hierarchical bayesian model, International Journal of Fatigue 117: 39-46. https: //doi.org/10.1016/j.ijfatigue. 2018.07 .027$.

MacKay, D. J. (1992). Bayesian interpolation, Neural computation 4(3): 415-447. https: //doi .org/10.1162/neco. 1992.4 .3 .415 .

Moreno, S. R., da Silva, R. G., Ribeiro, M., Fraccanabbia, N., Mariani, V. C. and Coelho, L. d. S. (2019). Very shortterm wind energy forecasting based on stacking ensemble, 14th Brazilian Computational Intelligence Meeting (CBIC), Belem, Brazil, pp. 1-7. https ://doi .org/10. 21528/CBIC2019-22.

Moreno, S. R., Mariani, V. C. and dos Santos Coelho, L. (2021). Hybrid multi-stage decomposition with parametric model applied to wind speed forecasting in brazilian northeast, Renewable Energy 164: 1508-1526. https://doi.org/10.1016/j.renene.2020.10.126.

Nelder, J. A. and Wedderburn, R. W. M. (1972). Generalized linear models, Journal of the Royal Statistical Society. Series A (General) 135(3): 370-384. https : //doi . org/10. $2307 / 2344614$. 
Persson, C., Bacher, P., Shiga, T. and Madsen, H. (2017). Multi-site solar power forecasting using gradient boosted regression trees, Solar Energy 150: 423-436. https: //doi.org/10.1016/j. solener.2017.04.066.

Ring, M. and Eskofier, B. M. (2016). An approximation of the gaussian rbf kernel for efficient classification with svms, Pattern Recognition Letters 84: 107-113. https: //doi.org/10.1016/j.patrec.2016.08.013.

Takeda, A. and Kanamori, T. (2014). Using financial risk measures for analyzing generalization performance of machine learning models, Neural Networks 57: 29-38. https://doi.org/10.1016/j. neunet. 2014.05.006.

Trierweiler Ribeiro, G., Guilherme Sauer, J., Fraccanabbia, N., Cocco Mariani, V. and dos Santos Coelho, L. (2020). Bayesian optimized echo state network applied to shortterm load forecasting, Energies 13(9): 2390. https:// doi.org/10.3390/en13092390.

Zeren, F. and Akkuş, H. T. (2020). The relationship between renewable energy consumption and trade openness: New evidence from emerging economies, Renewable Energy 147: 322-329. https://doi .org/10. 1016/j. renene. 2019.09.006. 